\title{
A Multistakeholder-Centric Data Analytics Governance Framework for Medication Adherence and Improvement in Rural Settings
}

\author{
Olawande Daramola ${ }^{1(\bowtie)}$ and Peter Nyasulu ${ }^{2}$ \\ ${ }^{1}$ Department of Information Technology, Cape Peninsula University \\ of Technology, Cape Town, South Africa \\ daramolaj@cput.ac.za \\ 2 Division of Epidemiology and Biostatistics, Faculty of Medicine and Health \\ Sciences, Stellenbosch University, Cape Town, South Africa \\ pnyasulu@sun.ac.za
}

\begin{abstract}
Good medication adherence is directly proportional to good health recovery and general improvement of a patient's health condition. Although many good medication adherence monitoring methods/techniques exist, the level of medication adherence for some chronic diseases by patients in rural settings is still suboptimal. Hence, the need for healthcare organisations to devise viable governance frameworks that will facilitate effective medication adherence monitoring and improved adherence by patients. This paper presents the conceptual overview of a governance framework for medication adherence monitoring and improvement that enables the collaboration of multiple stakeholders and data analytics (MUCODAF) in support of the patient in the treatment journey. The framework allows relevant stakeholders such as Healthcare workers (HCW), family members, and close friends to collaborate in support of a patient through the engagement of critical human factors such as empathy, motivation, encouragement, flexibility, and negotiation. The use cases of the framework, its technical composition, and the implementation plan are discussed in this paper. A concrete example of the application of the governance framework for medication adherence monitoring and improvement for a Tuberculosis patient in the African Country of Lesotho is presented to highlight the plausibility of the framework.
\end{abstract}

Keywords: Digital healthcare $\cdot$ Medication adherence $\cdot$ Data analytics $\cdot$ Smart wearables $\cdot$ Clinical governance $\cdot$ Data-driven decision making

\section{Introduction}

Generally, patients have a higher probability of recovery from chronic ailments if they keep to agreed medication schedules as prescribed by medical practitioners [1]. An optimal level of positive adherence behaviour is difficult to attain in low resource settings and rural areas due to many socio-economic problems. So far, several adherence-monitoring technologies such as Direct Observation Therapy (DOT), Video Observation Therapy (VOT), Indirect Monitoring Technology (Patient-facilitated or 
Device-facilitated), and Direct Monitoring Technology are being used to monitor adherence [2,3]. However, these methods have not yielded maximum improvement in treatment outcomes because of the challenges of medication adherence particularly associated with older people, and persons in rural settings [4]. The socio-economic challenges of people in the rural setting ranging from poverty, illiteracy, poor infrastructure, shortage of good medical facilities, and medical experts make it difficult for healthcare organisations to effectively monitor medication adherence of patients in these settings and to motivate them for good adherence behaviour. Generally, existing methods have failed to embrace a multiple stakeholder approach that can encourage the patient towards positive adherence during the treatment journey [3]. There is also a lack of consideration for the dynamics of the everyday life of a patient, which could affect adherence behaviour. A patient is adjudged to have negative adherence behaviour if there is no consistency in the timing, dosage, and frequency of taking medication, or there is an outright disregard for the agreed medication schedule. However, irregular adherence behaviour could be due to challenges of work, home, and other circumstances that obstruct a patient's normal schedule. The existing adherence monitoring methods have failed to include critical human factors such as motivation, encouragement, negotiation, and flexibility that recognise the dynamics of everyday life of a patient into the design of their medication adherence monitoring scheme, which limits their patient-centeredness. These adherence monitoring approaches have not made adherence improvement an integral part of their design in a way that is supportive of the patient. This paper presents the conceptual overview of a governance framework for medication adherence monitoring and improvement that will enable the collaboration of multiple stakeholders and data analytics (MUCODAF) to ensure that good decisions are made by the healthcare organisations when dealing with patients with different observed levels of medication adherence.

Relative to existing adherence monitoring methods, the proposed MUCODAF is conceived to embrace a more patient-centered perspective that makes key human factors such as motivation, encouragement, negotiation, and flexibility an integral part of the medication treatment adherence monitoring process through a multiple stakeholder approach. The composition of the framework is based on the integration of cloud computing, wearable sensors, mobile technology, artificial intelligence (AI), machine learning (ML), and data analytics. The governance framework creates a basis for intelligent and data-driven decision making by allowing relevant messages to be sent to the stakeholders from time to time based on the signals received from the wearable sensor device. Also, the post-analysis report of patients' adherence behaviours can be generated weekly by the healthcare workers (HCW) in healthcare organisations, which will provide a basis to determine the right strategy to deal with individual patients based on their identified adherence behaviours.

The rest of this paper is described as follows. Section 2 provides a review of the literature on medication adherence, while Sect. 3 presents a description of the proposed framework in terms of its core use cases, and its technical composition. In Sect. 4, an example of the application of the proposed framework for tuberculosis (TB) adherence monitoring and improvement is presented. The implementation plan and imperatives for the framework are discussed in Sect. 5, while the paper is concluded in Sect. 6 with a short note. 


\section{Literature Review}

Adherence monitoring approaches can be categorised into direct monitoring methods, and indirect monitoring methods. Direct monitoring methods involve directly observing the patient as the drug is taken or immediately by testing the body fluid of the patient in the laboratory. Indirect monitoring entails the use of technology devices for tracking activities that could signal when a patient has taken the drug or not. Many indirect adherence monitoring methods are device-facilitated by using various technologies [3, 4]. Device-facilitated measuring can be classified into various categories such as sensor-based systems, proximity-based systems, vision-based systems, and fusion-based systems [4]. The sensor-based system can be classified into three subclasses, which are smart pill containers, wearable sensors, and ingestible biosensors. The use of smart pill containers entails the detection of cap opening and bottle pick up to track adherence. Examples of these type of initiatives include Wisepills [5], Memscap [6], GlowCap [3, 7], Evrimed [8], and Amiko [9]. Wearable sensors detect motions related to cap twisting, hand-to-mouth, pouring the pill into the hand, and pill swallowing. Neck-worn sensors [10, 11], and wrist-worn sensors (in the form of smartwatches) [12-14] have been used to track medication adherence.

Also, ingestible biosensors have been used to detect pill ingestion by patients to track medication adherence [15, 16]. Currently, the use of ingestible biosensors has proved to be the most accurate, however, many patients will not find it convenient to ingest a biosensor so that they can be tracked to determine if they have taken their medication or not $[3,15,17]$. Proximity-based systems are non-invasive systems that detect medication presence or absence within the proximity of a reader's antenna by using computer vision technology. Vision-based systems detect medication presence or absence within the scope of the camera, while fusion-based systems try to verify the operation of monitoring the medication-taking activity [4]. All of these methods have been found to perform creditably well in tracking medication adherence with different levels of accuracy, but they are strictly medication adherence monitoring methods. They did not include medication adherence improvement as an integral part of their design as envisioned by our proposed framework in a way that offers support to the patient for improved adherence behaviour.

\section{Description of the Proposed Framework}

In this section, we present an overview of the affordances of the multistakeholder data analytics framework (MUCODAF) and the technical composition of the framework.

A governance framework for medication adherence and improvement is a system that makes a healthcare organization to be responsible, and accountable for:

- Continuously improving patients' medication adherence behaviours

- Maintaining a high level of medication adherence among patients

- Ensuring good treatment outcomes based on acceptable medication adherence behaviours. 


\subsection{Affordances of the Multistakeholder Collaborative Data Analytics Framework}

The proposed Multistakeholder Collaborative Data Analytics Framework (MUCODAF) affords five core use cases that pertain to medication adherence monitoring and improvement. These are described as follows.

Digital Collaboration of Multiple Stakeholders. MUCODAF will enable the exchange of messages between a specific stakeholder (patient supporter) and a patient, and a stakeholder and an HCW via a dedicated mobile app.

Description. Relevant stakeholders that have been enrolled with the consent of the patient to participate in the adherence monitoring process can communicate with a patient directly or share information with the HCW based on their observations on the adherence behaviour of the patient. A dedicated mobile app for adherence monitoring (to be developed) will be installed on a smartphone and other mobile devices for this purpose. The mobile app will enable all stakeholders to send messages to the patient, and the HCW by using four alternative means, which are app-to-app, the app to WhatsApp, the app to SMS, and app to email. A patient supporter that has only a cellphone (not a smartphone) can send an SMS or make a call to the HCW on their observations of the adherence behaviour of the patient. For SMS, this can be sent via a specific code to the mobile app of the HCW. The essence of the specific code is to ensure that such SMS messages are free of charge or at a subsidized rate. Government and other donor agencies could come in to have special arrangements with telecommunication/mobile service providers to support the patients with chronic ailments and in need of critical care in this way. Disease such as Cancer, Tuberculosis, HIV, and Diabetes are prominent in developing countries where this kind of governmental and external support for patients will be useful. A stakeholder (patient supporter) may also prefer to use alternative means of communication such as email that is sent from a desktop or laptop to contact the HCW.

Medication Adherence Monitoring. MUCODAF will enable continuous acquisition of data on specific events that suggest adherent behaviour of the patient through a smartwatch and a mobile device. The information is subsequently stored in a cloud database and also transmitted to relevant stakeholders.

Description. The framework will enable medication adherence monitoring through a coordinated operation that will involve the use of an outdoor, waterproof smartwatch that is worn by the patient. The smartwatch is paired with the smartphone of the patient via Bluetooth Low Energy (BLE) connectivity to ensure that information is sent regularly to a cloud database via $\mathrm{Wi}-\mathrm{Fi}$ or $2 \mathrm{G} / 3 \mathrm{G} / 4 \mathrm{G}$ data transmission. Adherence information is stored locally on the smartphone until a period when the patient has access to WiFi or mobile data when the stored information is transferred into the cloud. Medication adherence tracking with smartwatch will be done by sensing the Near Field Communication (NFC) tag on the drug casing. When the patient opens the drug box, he receives a message asking to confirm if he has taken his drug, if the reply is a yes, the event is recorded as an instance of positive adherence. A timestamped record of the event is stored on his phone for subsequent transfer to the cloud database. From the 
stored data, a patient supporter who is co-monitoring the adherence of the patient receives a message that the patient has taken his drug for that day. At the end of the week, the patient supporters (stakeholder, HCW, etc.) also get an adherence activity report via the mobile app/email/WhatsApp on the level of adherence of the patient in terms of compliance with the prescribed/agreed medication schedule for that week. Negotiation comes in when a patient requests an adjustment to a previously agreed schedule due to changes in his daily activities which may affect his adherence to an existing schedule. A patient can request adjustment of an existing medication schedule by sending a message to the HCW from the mobile app. Once a negotiated and revised schedule has been approved for the patient, it then becomes the basis for monitoring the patient's adherence by all stakeholders. A cloud-based central coordinating service ensures that information on a patient's adherence is sent through various channels such as SMS, WhatsApp, or email depending on the preferred mode of communication of a patient supporter. The patient supporter will use the available information on the adherence behaviour of a patient to determine how best to support the patient by way of encouragement, advice, or commendation to improve the level of adherence. The patient supporter is also able to contact the HCW when it is deemed necessary.

Data-Driven Decision Making. MUCODAF will facilitate a decision support system for HCW via the Mobile App based on the analysis of medication adherence data. By engaging data analytics services through Machine learning, and intelligent recommendations, the HCW can make informed decisions on the treatment of a patient.

Description. The framework will support various AI-based, and data analytics services such as predictive analytics, descriptive analytics, diagnostic analytics, and prescriptive analytics based on the data that have been acquired over time. These operations will mostly be executed using the backend resources of the framework, but the user will access the results/reports of the analytic services from their mobile apps. By using machine learning and statistical learning algorithms, periodic reports can be generated on a patient's adherence behaviour on a weekly and monthly basis. The report of these analyses will be accessible by HCW via an interface of the adherence monitoring mobile app. It will be possible for an HCW (Doctor, nurse) to obtain reports on adherence of a specific patient, or group of patients or run specific queries based on an issue of interest. Reports on specific patient groups/categories and selected demographics can also be generated from the framework on a monthly, quarterly, biannually or yearly basis for decision making at higher levels of healthcare management, and policy formulation.

Personalised Treatment Strategy. MUCODAF will foster decision support for HCW through intelligence recommendations. It will ensure a review of the patient's treatment regimen by $\mathrm{HCW}$.

Description. The intelligent analysis of medication adherence data through various analytics methods will make it possible for the HCW (Doctor, Nurse) to devise the most appropriate way to engage with a patient to ensure a good treatment outcome. This may include scheduling counselling sessions for patients that are found to be nonadherent, and individual follow-up sessions in some cases to ensure improvement in a patient's adherence behaviour. It will also be possible to receive intelligent 
recommendations as a form of decision support on the course of action to deal with a patient based on the report of the adherence behaviour and the state of his health after clinical examination.

Adherence Behaviour Data Archiving and Curation. MUCODAF will allow automated processing of data in a way that ensures archiving and curation of data into relevant categories and classes for reuse.

Description. The framework will enable data on adherence behaviour that has been collected over time to be categorized and allotted to various classes and clusters. This will aid data retrieval and data reuse particularly for planning and policy formulation by healthcare organizations, and the national government.

The technical composition of the MUCODAF comprising a smartphone, smartwatch, Bluetooth (BLE), and IoT Hub is shown in Fig. 1, while the mapping of the use cases to specific actors in terms of the initiator of an action, the receiver/beneficiaries of such a use case, is shown in Table 1. The BLE ensures that there is a continuous connection between the smartwatch, and the patient's mobile devices as long as both devices are on. The only concern is the need to ensure that the devices are charged regularly. Recent advancement in technology ensures that wearable devices are now able to work for longer periods before it is necessary to recharge their batteries. This explains why an increasing number of medication adherence methods are based on the wearable technologies that use sensors $[3,4]$.

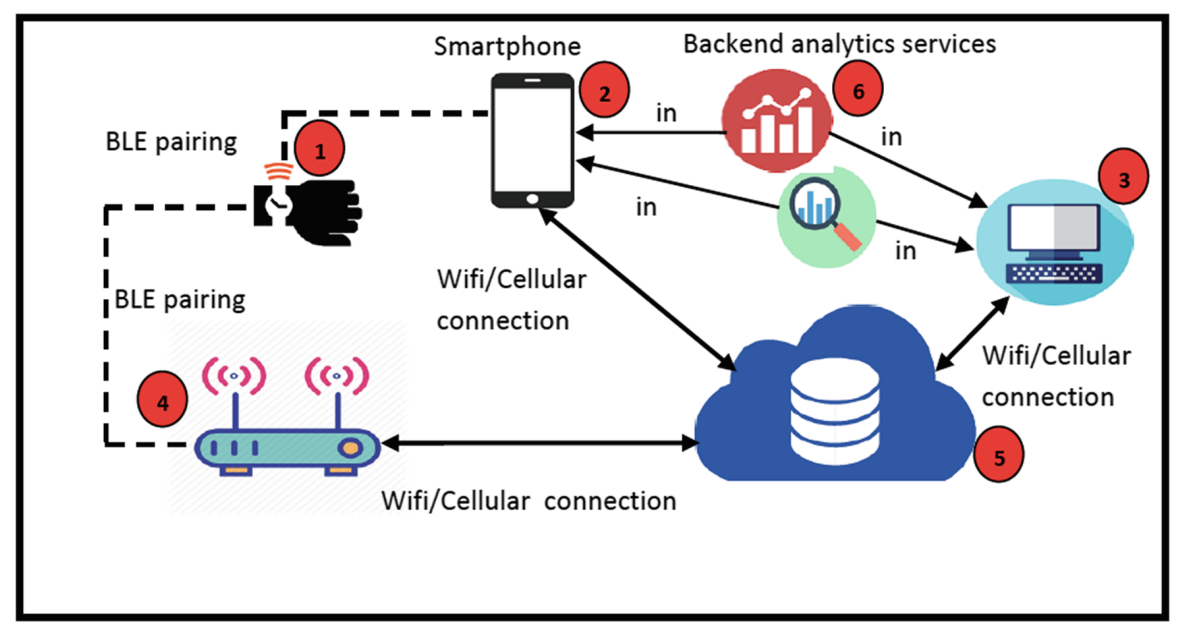

Fig. 1. An overview of the composition of the MUCODAF for improved medication adherence

\subsection{Potential for Generalization of the MUCODAF}

The selected use cases that are presented are not exhaustive, but those considered as basic to ascertain the plausibility of the proposed MUCODAF framework. It is quite possible that additional valid use cases that pertain to medication adherence and 
Table 1. Use case mapping of affordances to specific actors

\begin{tabular}{|c|c|c|}
\hline $\mathrm{S} / \mathrm{n}$ & Affordance & Description \\
\hline 1 & $\begin{array}{l}\text { Digital collaboration of } \\
\text { multiple stakeholders }\end{array}$ & $\begin{array}{l}\text { Receiver/Beneficiary } \\
\text { Patient: motivation, encouragement, negotiation, } \\
\text { flexibility } \\
\quad \text { HCW: timely update on the patient's progress }\end{array}$ \\
\hline 2 & $\begin{array}{l}\text { Medication adherence } \\
\text { monitoring }\end{array}$ & $\begin{array}{l}\text { Initiator } \\
\text { Patient: initiates action } \\
\text { Wearable device: smartwatch } \\
\text { Mobile device: smartphone, cellphone } \\
\text { Software: cloud-based Adherence monitoring service } \\
\text { Receiver/Beneficiary } \\
\text { Stakeholders: HCW, friends, family member }(s) \text { : } \\
\text { timely update on patient's medication adherence }\end{array}$ \\
\hline 3 & Data-driven decision making & $\begin{array}{l}\text { Initiator } \\
\text { HCW: takes decision-based on analysis of gathered } \\
\text { data, and observed patterns } \\
\text { Health Sector: take decisions based on generally } \\
\text { observed patterns on adherence behaviour for various } \\
\text { patients } \\
\text { Government: make plans and policies regionally, and } \\
\text { nationally } \\
\text { Receiver/Beneficiary } \\
\text { Patients: improved healthcare } \\
\text { HCW (Doctors, Nurses, etc.): decision support for } \\
\text { improved service delivery } \\
\text { Health Sector: data for improvement of healthcare } \\
\text { services } \\
\text { Government: data for planning and development } \\
\text { Society: a healthier society, improved healthcare }\end{array}$ \\
\hline 4 & $\begin{array}{l}\text { Personalised treatment } \\
\text { strategy }\end{array}$ & $\begin{array}{l}\text { Initiator } \\
\quad H C W \text { : devise person-specific methods and strategies } \\
\text { to help a patient based on the observed adherence } \\
\text { behaviour } \\
\text { Receiver/Beneficiary } \\
\quad \text { Patient: receives the right attention and care that is } \\
\text { necessary } \\
\quad H C W \text { : improved success rate in healthcare }\end{array}$ \\
\hline 5 & $\begin{array}{l}\text { Adherence behaviour data } \\
\text { archiving and curation }\end{array}$ & $\begin{array}{l}\text { Initiator } \\
\quad M U C O D A F \text { : an automated process of storing } \\
\text { relevant data for future use } \\
\text { Receiver/Beneficiary } \\
\quad \text { Health Sector: data-driven process improvement in } \\
\text { the healthcare sector } \\
\quad \text { Government: data stored will be used for policy } \\
\text { development. Statistics }\end{array}$ \\
\hline
\end{tabular}


improvement be can yet be identified in the circumstances of an actual implementation. Therefore, the design of the MUCODAF should be based on a modular architecture that will ensure that additional use cases other than the basic ones highlighted in Sect. 3.1 can be catered for. Thus, with the choice of a modular design, it will be possible to adapt the MUCODAF to suit different contexts on the issue of medication adherence and adherence improvement.

\section{Tuberculosis (TB) Medication Adherence with MUCODAF}

In this section, we demonstrate by using textual narratives how MUCODAF can be applied to the case of a Tuberculosis (TB) patient under care in need of adherence monitoring. The narrative provided embraces key aspects of the 5 main use cases of MUCODAF.

\subsection{Background of the TB Patient in Lesotho}

As extracted from [18], Mr. T is a chronic TB patient (age - 45 years old) that works in a major mine in Lesotho. He lives with his wife and four children in a mining community/township that is called Botha Bothe in Lesotho. Mr. T has been enrolled for $\mathrm{TB}$ treatment for six months in the local hospital in Botha Bothe and he is expected to take his medication twice a day at $6 \mathrm{~h}$ interval. Mr. T has agreed with the healthcare worker that he will take his drug at 7 am every morning and at $1 \mathrm{pm}$ during the lunch break while at work, and maintain the same schedule on non-work days. The medication schedule was agreed with the healthcare worker in the presence of the wife of Mr. T (Mrs. T) and Joe, who is a close friend and associate of Mr. T at work. Mrs. $\mathrm{T}$ and Joe have been enrolled as co-monitors and patient supporters to aid Mr. T in the aspect of adherence to the agreed schedule. A challenge with Mr. T is that lunch breaks at work do vary at times depending on the work schedule. For example, there are times when Mr. T and his colleagues need to spend longer periods in the mine, which would require that lunchtime be postponed until a later time.

\subsection{Scenario Description of MUCODAF at Work}

To apply the MUCODAF, there must be a centralized cloud-based coordination service that runs 24/7. The coordination service should be able to send messages to a patient through a wearable device (smartwatch, smartphone) when it is required, and periodically to all the patient supporters (stakeholders). With this in place, the following scenario can then be realized [18]:

1. The smartwatch device alerts Mr. T 15 min to the time when his drug is due to be taken.

2. At the specified time, the smartwatch starts to make an alarm sound.

3. The smartwatch tracks the medication adherence event by sensing the NFC (Near Field Communication) tag on the drug casing and prompts Mr. $\mathrm{T}$ if indeed he has taken his drug. 
4. If Mr. T answers in the affirmative, then data consisting of the time when the drug was taken, location, date, and a score that is indicative of a positive adherence behaviour of Mr. T ("Yes" - positive; "No" - negative) is stored in the cloud database. If there is internet connectivity the data is sent directly to the cloud through the local IoT hub. If there is no connectivity, the data is stored temporarily on Mr. T's smartphone, and sent subsequently to the cloud (this is because the patient's smartphone that has been paired with the wearable smartwatch via BLE).

5. A reminder message is sent to $\mathrm{Mr}$. $\mathrm{T}$. via his smartphone and smartwatch every $10 \mathrm{~min}$ within $30 \mathrm{~min}$ from when he is supposed to have taken his medication to motivate him to take his medication according to the current medication schedule. If a message of positive adherence is not received $45 \mathrm{~min}$ after the expected time, then a conclusion of nonadherence is made by the system.

6. A daily report that conveys the adherence behaviour of $\mathrm{Mr}$. $\mathrm{T}$ (positive/negative) is sent to Mrs. T, Joe, and $\mathrm{HCW}$. The HCW receives a more detailed report that shows the specific time, date, and place when the drug was taken, and times when medications were missed.

7. Mr. T. is allowed to change the time of his medication (possibly due to workrelated, family, emergency, ad hoc events), once a week through the negotiation module of the mobile app. Once a new medication schedule has been selected, this communicated to all stakeholders. The newly negotiated schedule then becomes active from that point forward as the new treatment adherence plan that will be monitored by stakeholders.

8. After a new negotiated treatment plan has been selected by Mr. T., it is then used subsequently by the cloud-based coordination service to send messages to the smartwatch and the smartphone of Mr. T, and other stakeholders.

9. The suite of cloud-based data analytics services that can be accessed from the dedicated mobile app for adherence monitoring is used by the HCW to generate a report on the adherence behaviour of a patient periodically, which could lead to a personalised and differentiated engagement with a TB patient to improve his adherence behaviour.

10. All stakeholders can exchange messages or chat on issues that pertain to the TB patient to which they are affiliated via the mobile app, WhatsApp or email.

\section{Implementation Plan for MUCODAF}

To ascertain the viability of the MUCODAF, it is essential to demonstrate its application in a real study context. In this section, we discuss the imperatives for the concrete implementation of MUCODAF. This will involve several aspects such as recruitment of participants, selection of the study cites, system development, and training of the participants. These salient aspects are discussed below.

Setting. To assess the plausibility of our proposed approach, a study site called Botha Bothe in Lesotho has been selected. The consent of a local hospital has been secured, while ethical clearance received from the Department of Health in Lesotho, and the two universities that are involved in the research (the university affiliations of the authors of 
this paper). A cohort study involving a few patients would be undertaken in the first instance (there is a plan to 20-40 patients), which would later be scaled up with more subject when more funding is received. Each of the patients will be provided with a smartwatch, and a smartphone, while a specific patient supporter will also be assisted with appropriate technology devices that would make it possible for them to play their co-monitoring role effectively.

System Development. A mobile app that is dedicated to adherence monitoring shall be provided for the study. The key features of the mobile app will include capability to enable patients, and patient supporters' participant to send messages to the HCW, $\mathrm{HCW}$ being able to send messages to relevant persons, $\mathrm{HCW}$ receiving intelligent recommendations, and being able to view data analytics results (predictive, prescriptive, diagnostic, descriptive) for decision making. An overview of the key features of the mobile app is shown in Fig. 2.

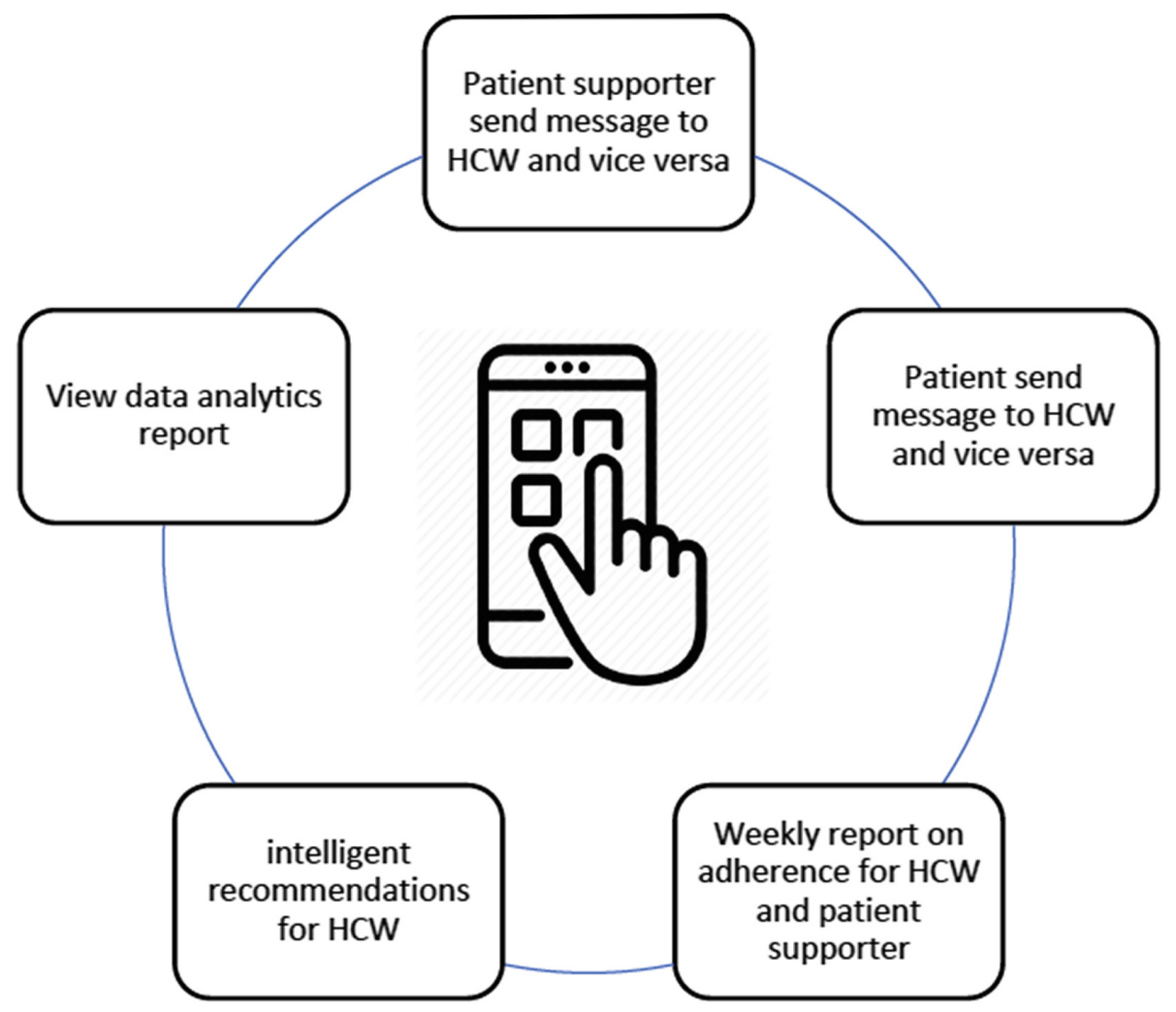

Fig. 2. Overview of features of the mobile app for medication adherence

Training Participants. All participants in the proposed study have to be trained on the use of medication adherence monitoring mobile app, and the smartwatch and smartphone. This is particularly important because the subjects in the study would mostly the 
less educated persons that not technically savvy. This makes it necessary to conduct orientation and training sessions for them. The training will include all HCWs, patients, and patient supporter that have been selected to participate in the cohort study.

Support of Government and Relevant of Donor Agencies. The conceptualization of the MUCODAF is meant to elicit the support of relevant government agencies and donors who are committed to improved healthcare of the less privileged people. The practicality of MUCODAF for improving medication adherence will require that a patient be supported by providing smartwatches, and smartphones for them. Donor agencies may also need to offer free internet/mobile data packages for a patient and the patient supporters that have been enrolled to co-monitor adherence of a patient at least for the period when the patient is under medication.

\section{Conclusion}

In this paper, we have presented the concept of a multistakeholder collaborative data analytics framework for improved medication adherence (MUCODAF). In contrast to existing medication adherence monitoring approaches, most of which focus mainly on adherence tracking and measurement, MUCODAF seeks to integrate both medication adherence and adherence improvement of the patient. To achieve this, the notion of multiple stakeholder collaboration that is driven by sensor-based adherence tracking and data analytics was introduced to support the patient in the treatment journey. Although MUCODAF is still at the conceptual stage, the imperatives for its implementation were also discussed, as well as the plausibility of its adoption for medication adherence monitoring and improvement in low resource settings. Our immediate plan is to commence the execution of the MUCODAF based on the research fund that is currently available to us. Thereafter, an evaluation in terms of the usability of the framework from the perspective of the participants will be undertaken, and its effectiveness to monitor medication adherence and endanger improvement medication adherence.

\section{References}

1. Alipanah, N., et al.: Adherence interventions and outcomes of tuberculosis treatment: a systematic review and meta-analysis of trials and observational studies. PLoS Med. 15(7), e1002595 (2018)

2. UNAIDS. UNAIDS Global Report 2016. www.unaids.org/sites/default/files/media_asset/ global-AIDS-update-2016_en.pdf. Accessed 29 Feb 2019

3. DiStefano, M., Schmidt, H.: mHealth for tuberculosis treatment adherence: a framework to guide ethical planning, implementation, and evaluation. Glob. Health: Sci. Pract. 4(2), 211-221 (2016)

4. Aldeer, M., Javanmard, M., Martin, R.: A review of medication adherence monitoring technologies. Appl. Syst. Innov. 1(2), 14 (2018)

5. Haberer, J., et al.: Real-time adherence monitoring of antiretroviral therapy among HIVinfected adults and children in rural Uganda. AIDS (London, England) 27(13), 2166-2168 (2013) 
6. Byerly, M., et al.: A comparison of electronic monitoring vs. clinician rating of antipsychotic adherence in outpatients with schizophrenia. Psychiatry Res. 133(2-3), 129-133 (2005)

7. Kalantarian, H., Alshurafa, N., Nemati, E., Le, T., Sarrafzadeh, M.: A smartwatch-based medication adherence system. In: 2015 IEEE 12th International Conference on Wearable and Implantable Body Sensor Networks (BSN), pp. 1-6. IEEE (2015)

8. Sumari-de Boer, M., et al.: Implementation and effectiveness of evriMED with short messages service (SMS) reminders and tailored feedback compared to standard care on adherence to treatment among tuberculosis patients in Kilimanjaro, Tanzania: proposal for a cluster randomized controlled trial. Trials 20(1), 426 (2019)

9. Braido, F., Paa, F., Ponti, L., Canonica, G.: A new tool for inhalers' use and adherence monitoring: the Amiko® validation trial. Int. J. Eng. Res. Sci. 2(10), 159-166 (2016)

10. Kalantarian, H., Alshurafa, N., Le, T., Sarrafzadeh, M.: Non-invasive detection of medication adherence using a digital smart necklace. In: Proceedings of the IEEE International Conference on Pervasive Computing and Communication Workshops (PerComWorkshops), St. Louis, MO, USA, 23-27, pp. 348-353 (2015)

11. Kalantarian, H., Alshurafa, N., Sarrafzadeh, M.: A survey of diet monitoring technology. IEEE Pervasive Comput. 16, 57-65 (2017)

12. Putthaprasat, T., Thanapatay, D., Chinrungrueng, J., Sugino, N.: Medicine intake detection using a wearable wrist device accelerometer. In: Proceedings of the International Conference on Computer Engineering and Technology, Bangi, Malaysia, 4-5 December, vol. 40, pp. 15 (2012)

13. Bennett, J., Rokas, O., Chen, L.: Healthcare in the smart home: a study of past, present, and future. Sustainability 9, 840 (2017)

14. Chen, H., Xue, M., Mei, Z., Bambang Oetomo, S., Chen, W.: A review of wearable sensor systems for monitoring body movements of neonates. Sensors 16, 2134 (2016)

15. Hafezi, H., Robertson, T.L., Moon, G.D., Au-Yeung, K.Y., Zdeblick, M.J., Savage, G.M.: An ingestible sensor for measuring medication adherence. IEEE Trans. Biomed. Eng. 62, 99-109 (2015)

16. Kalantar-Zadeh, K., et al.: A human pilot trial of ingestible electronic capsules capable of sensing different gases in the gut. Nat. Electron. 1, 79-87 (2018)

17. Chai, P., Rosen, R., Boyer, E.: Ingestible biosensors for real-time medical adherence monitoring: MyTMed. In: Proceedings of the Annual Hawaii International Conference on System Sciences, vol. 2016, p. 3416 (2016)

18. Daramola, O., Nysaulu, P.: A digital collaborative framework for improved tuberculosis treatment adherence of patients in rural settings. In: 2019 Open Innovations (OI), pp. 297303. IEEE (2019) 